\title{
Wirtschaftlichkeitsbonus beim Labor wird reformiert
}

_ Aktuell erhalten Hausärzte einen Bonus für nicht erbrachte bzw. veranlasste Laborleistungen über die von der $\mathrm{KV}$ automatisch zugesetzte Nr. 32001 EBM. Die Zuteilung hängt davon $\mathrm{ab}$, ob ein sehr kompliziert berechnetes fachgruppenspezifisches Laborbudget überschritten wird. Die KBV will diese Berechnung ab Juli 2017 vereinfachen und die Zahlung des Wirtschaftlichkeitsbonus in einer Art Drei-Stufen-Regelung modifizieren.

Zunächst wird für die Praxis jedes Quartal ermittelt, wie hoch die Laborkosten pro kurativ-ambulantem Behandlungsfall sind. Berücksichtigt werden dabei alle bezogenen, als Auftragsleistung überwiesenen oder selbst erbrachten Untersuchungen aus den EBMAbschnitten 32.2 und 32.3. Nicht einbezogen werden auch weiterhin die Leistungen aus der Ausnahmeliste. Die Liste wird bei der Gelegenheit übrigens auch überarbeitet.

Nun wird dieser praxisspezifische Fallwert mit der gesamten Arztgruppe verglichen. Hier gibt es eine Spanne zwischen einem unteren und einem oberen
Fallwert. Trifft eine Praxis mit ihren Laborkosten den unteren Fallwert oder unterbietet ihn sogar, wird die Nr. 32001 in voller Höhe bezahlt. Ab dem oberen Fallwert wird die Nr. mit 0 Punkten bewertet. Liegt er irgendwo dazwischen, wird die Bewertung abgestaffelt, und zwar prozentual, je nach Position des Fallwerts zwischen den Grenzwerten.

\section{MMW-KOMMENTAR}

Die Neuerung wird zweifelsohne die KV-Verwaltung entlasten. Die KBV hofft allerdings auch, das Wirtschaftlichkeitsbewusstsein der Vertragsärzte zu verbessern - und damit den inflationsartigen Anstieg der Laborkosten zu bremsen oder gar zu stoppen. Ob diese Rechnung aufgeht, ist fraglich - es sei denn, die Maßnahme würde mit einer massiven Aufklärungsaktion verbunden, die dem einzelnen Arzt aufzeigt, wie sein persönliches Honorar steigt, wenn er weniger Laborleistungen veranlasst. Die Praxislaborleistungen nach den Nrn. 32 125, 32880, 32881 und 32882 werden übrigens ausdrücklich aus der Berechnung ausgenommen und führen damit nicht zu einer Minderung des Honorars beim Wirtschaftlichkeitsbonus.

\section{Beispiel: So soll der Bonus errechnet werden}

Für die Allgemeinärzte gibt die KV einen arztgruppenspezifischen Fallwert in Punkten an. Der untere Wert sei 18 Punkte pro Behandlungsfall, der obere Fallwert 39 Punkte.

Eine Hausarztpraxis habe nun in einem Quartal 1.000-mal die Versichertenpauschale abgerechnet. Wenn sie im Schnitt höchstens Laborleistungen für 18 Punkte abgerechnet hat, insgesamt also höchstens für 18.000 Punkte, wird das Honorar für den Wirtschaftlichkeitsbonus in voller Höhe ausbezahlt. Mo- mentan ist für die Bonus-Nr. 32001 eine Bewertung von 24 Punkten vorgesehen, das Honorar läge also bei 24.000 Punkten oder aktuell 2.527,20 Euro.

Wenn die Praxis Laborleistungen für 39.000 oder mehr Punkte abgerechnet hat, erhält sie dagegen kein Honorar für die Nr. 32001.

Liegt ihr Fallwert dazwischen, erhält sie einen prozentualen Anteil der möglichen Honorierung - je nachdem, wie weit sie sich vom Fallwert 18 entfernt. 